\title{
Recurrent Extraskeletal Osteosarcoma
}

National Cancer Institute

\section{Source}

National Cancer Institute. Recurrent Extraskeletal Osteosarcoma. NCI Thesaurus. Code C8811.

The reemergence of extraskeletal osteosarcoma after a period of remission. 